
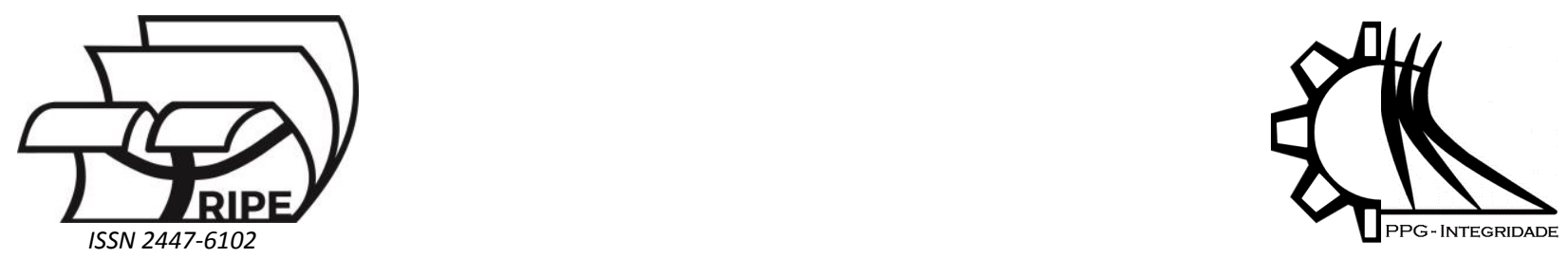

Article

\title{
Verificação de modelo computacional para placas com enrijecedores considerando condição de contorno de simetria
}

\author{
Amaral, R.R. ${ }^{1, *}$, Troina, G. da S. ${ }^{2}$, Cunha, M. L. ${ }^{3}$, Rocha, L.A.O. ${ }^{4}$, dos Santos, E.D. ${ }^{5}$ and Isoldi, L.A. ${ }^{6}$ \\ 1 Mestrando em Modelagem Computacional. Programa de Pós-Graduação em Modelagem Computacional (PPGMC) da \\ Universidade Federal do Rio Grande (FURG) - Rio Grande/RS; rodrigo_amaral_23@hotmail.com \\ 2 Mestre em Engenharia Oceânica. Programa de Pós-Graduação em Engenharia Oceânica (PPGEO) da FURG - Rio Grande/RS; \\ gregori.troina@gmail.com \\ 3 Graduando em Engenharia Mecânica Empresarial. Escola de Engenharia da FURG - Rio Grande/RS.; \\ marcelolamcunha@hotmail.com \\ 4 Doutor em Engenharia Mecânica. Professor do Programa de Pós-Graduação em Engenharia Mecânica da Universidade do Vale \\ do Rio dos Sinos (Unisinos) - São Leopoldo/RS; luizor@unisinos.br \\ 5 Doutor em Engenharia Mecânica. Professor do PPGEO e PPGMC da EE da FURG - Rio Grande/RS; elizaldosantos@furg.br \\ 6 Doutor em Engenharia Mecânica. Professor do PPGEO e PPGMC da EE da FURG - Rio Grande/RS; liercioisoldi@furg.br \\ * Correspondence: rodrigo_amaral_23@hotmail.com;
}

Received: 14/12/2018; Accepted: 05/01/2019; Published: 29/01/2019

Resumo: $O$ presente artigo apresenta a modelagem computacional de placas enrijecidas submetidas a uma carga transversal uniformemente distribuída, permitindo a análise numérica de deslocamentos e tensões. $O$ modelo computacional foi desenvolvido no software ANSYS, baseado no Método dos Elementos Finitos (MEF), empregando o elemento finito SHELL281. Este modelo foi elaborado considerando a condição de contorno de simetria, possibilitando que somente um quarto da placa enrijecida fosse simulado numericamente. As simulações com esta condição de contorno demonstraram um ganho de processamento computacional em relação às simulações da placa completa. Além disso, a sua utilização possibilitou o uso de discretizações espaciais (malhas) mais refinadas do que as usadas em modelos que consideram a placa inteira. Com o objetivo de verificar esse modelo computacional assim como avaliar sua acurácia em relação aos resultados obtidos com o modelo que considera toda a placa, análises de deflexão e de tensão foram realizadas. A verificação do modelo foi feita usando a solução independente de malha, obtida através de um teste de convergência de malha. Os resultados do modelo numérico utilizando a condição de contorno de simetria demonstraram uma boa precisão em relação ao modelo computacional da placa completa, tanto na verificação quanto em um comparativo da placa inteira em relação a seu equivalente com simetria.

Palavras-chave: Deflexão. Tensão. Verificação. Placa enrijecida. Enrijecedor.

\section{Computational model verification for plates with stiffeners considering symmetry boundary condition}

Abstract: The present article presents a computational modelling of stiffened plates submitted to a uniformly distributed transversal load, allowing the numerical analysis of displacements and stress. The computational model was developed in the ANSYS software, based on Finite Element Method (FEM), using the SHELL281 finite element. This model was elaborated considering the symmetry boundary condition, allowing only one quarter of the stiffened plate to be numerically simulated. The simulations with this boundary condition demonstrated a computational processing gain in relation to the complete plate simulations. Besides, its use enabled the employment of more refined spatial discretization (meshes) than those used in models that consider the entire plate. In order to verify this computational model as well as to evaluate its accuracy in relation to the results obtained with the model that considers the whole plate, deflection and stress analysis were performed. The verification of the model was done using the independent mesh solution, obtained through a mesh convergence test. The results of the numerical 
model using the symmetry boundary condition demonstrated good accuracy over the whole plate computational model, both in the verification and in a comparative of the whole plate in relation to its equivalent with symmetry.

Keywords: Deflection. Stress. Verification. Stiffened plate. Stiffener.

\section{Introdução}

Os engenheiros estruturais vem há muito tempo buscando a concepção de estruturas mais esbeltas que resistam a elevados carregamentos. De modo que, com o desenvolvimento de novos materiais e processos computacionais mais rápidos, uma nova fronteira foi aberta para a concepção e o desenvolvimento de ferramentas, técnicas, métodos e sistemas numéricos que atendessem esta otimização geométrica cada vez mais requisitada (Vellasco et al., 2014).

Um material bastante utilizado, graças a sua alta resistência nos diversos estados de tensão (tração, compressão e flexão), é o aço. A aplicação deste pode ser realizada através de placas ou painéis que são encontrados na construção civil, elaboração de estruturas de navios ou em estruturas aeroespaciais. Além disso, as placas podem ter sua rigidez aumentada através da soldagem de enrijecedores transversal e/ou longitudinalmente (Bellei, 1998).

Neste sentido o MEF tem sido empregado em diversos estudos de placas com enrijecedores, dentre os quais podem ser citados: Rossow \& Ibrahimkhail (1978) por desenvolver uma análise de placas enrijecidas concêntricas e excêntricas através da utilização do Método da Restrição em conjunto com o MEF; Salomon (2001) analisou e propôs diversas formas de como modelar placas enrijecidas através de modelos computacionais baseados no MEF; e, Troina (2017) apresentou uma otimização geométrica pelo Método do Design Construtal, via Busca Exaustiva, de modelos computacionais de placas enrijecidas submetidas a carregamentos transversais desenvolvidas no software ANSYS.

Considerando que a maioria das pesquisas adota a modelagem computacional da placa completa, o presente artigo tem como objetivo desenvolver um modelo numérico para placas enrijecidas empregando a condição de contorno de simetria. Ademais, será apresentada a verificação e um teste de convergência de malha que comparará os resultados de deflexão e tensão de flexão nos enrijecedores da placa inteira em relação a um quarto de placa com a condição de contorno de simetria.

Cabe destacar que o uso da condição de contorno de simetria permite reduzir o tempo de processamento e a utilização de uma malha mais refinada, uma vez que é possível considerar um domínio computacional com metade ou um quarto do problema quando este apresentar características de simetria em relação à geometria, ao carregamento, às condições de vinculação e às propriedades do material. A simetria, quando aplicada, faz com que os deslocamentos fora do plano e as rotações no plano sejam definidos como zero, isto é, quando esta condição de contorno se encontra paralela ao eixo $y$, os deslocamentos e rotações sofridas em $x$ devem ser nulas no local de sua aplicação, e quando ela se encontra paralela ao eixo $x$, os deslocamentos e rotações em $y$ que são nulos (ANSYS, 2017).

\section{Modelagem Computacional}

Um dos principais objetivos de uma modelagem numérica de estruturas de aço consiste em, depois de devidamente verificadas ou validadas, expandir o banco de dados referente a um determinado comportamento estrutural. A expansão é feita por meio de uma análise paramétrica, que possibilita a redução do tempo e custo da solução quando comparados com ensaios de laboratório convencionais (experimentos). Apesar disso, alguns cuidados devem ser tomados na especificação do escopo dessas análises, pois se elas foram calibradas para uma dada faixa de variação de suas principais variáveis controladoras, análises paramétricas fora destes limites serão sempre questionadas e muitas vezes invalidadas (Vellasco et al., 2014).

O emprego da modelagem computacional apresentada no presente trabalho ocorreu pelo software ANSYS, conforme pode ser observado na Fig. 1. Na Fig. 1(a) é apresentado o domínio computacional discretizado de uma placa quadrada com enrijecedores nas duas direções. Já na Fig 1(b) é mostrado o domínio computacional discretizado considerando condições de contorno de simetria, ou seja, somente 1/4 da placa da Fig. 1(a) é necessário. 


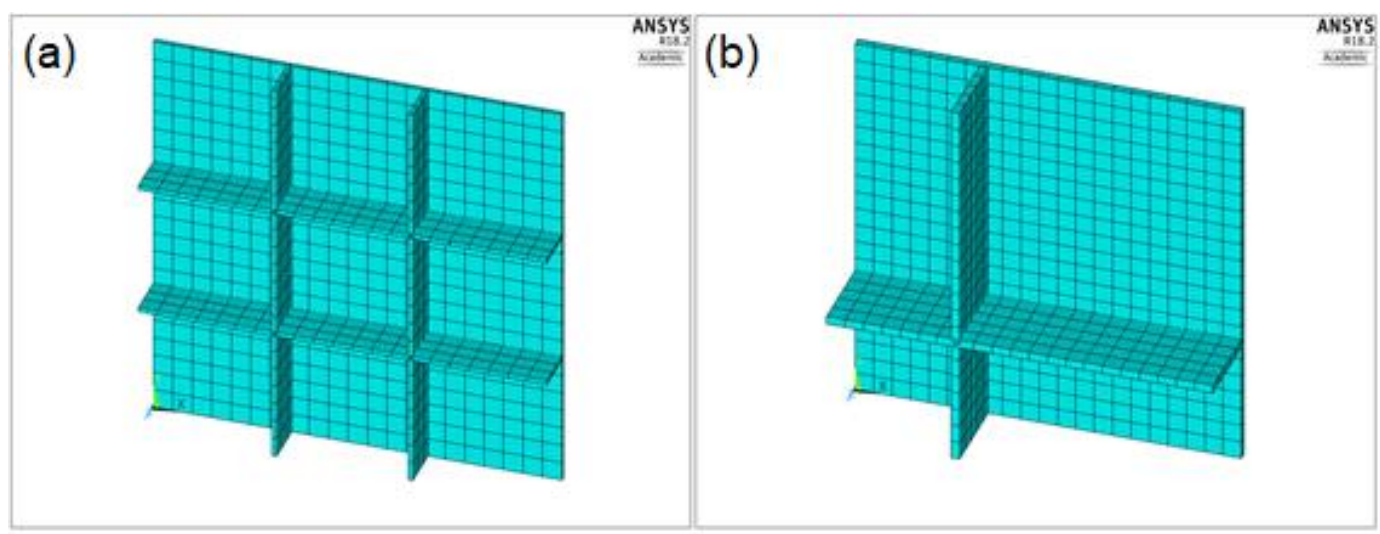

Figura 1. Domínios computacionais da placa completa e seu equivalente de um quarto com simetria.

O ANSYS é baseado no MEF e utilizado para resolver numericamente uma ampla variedade de problemas mecânicos. Podendo envolver: análises estruturais estáticas ou dinâmicas (ambas lineares ou não lineares); transferência de calor ou de fluido; problemas de acústica; problemas eletromagnéticos; entre outros (Stolarski \& Yoshimoto, 2018).

O MEF, por sua vez, pode ser definido pela discretização de um modelo matemático em um número finito de partes (elementos), cuja representação da geometria, condição de contorno e da carga imposta ao modelo são mantidas conforme o problema físico proposto. A sua solução só ocorrerá a partir da montagem da matriz global dos elementos que permitirá a solução nodal e, por consequência, a obtenção dos valores de deslocamento e tensão sofridos por cada elemento. Para casos que envolvam problemas de comportamento linear elástico, a matriz global é representada pela Eq. (1):

$$
\left\{\begin{array}{c}
q_{1} \\
q_{2} \\
\vdots \\
q_{m}
\end{array}\right\}=\left[\begin{array}{cccc}
K_{11} & K_{12} & \cdots & K_{1 m} \\
K_{21} & \ddots & & \vdots \\
\vdots & \vdots & & \vdots \\
K_{m 1} & \cdots & \cdots & K_{m m}
\end{array}\right]\left\{\begin{array}{c}
u_{1} \\
u_{2} \\
\vdots \\
u_{m}
\end{array}\right\}+\left\{\begin{array}{c}
f_{1} \\
f_{2} \\
\vdots \\
f_{m}
\end{array}\right\}
$$

onde $\vec{q}$ representa o vetor da força, $m$ é o número de componentes de força a serem consideradas em cada nó, $|K|$ é a matriz de rigidez, $\vec{u}$ é o vetor dos deslocamentos nodais e $\vec{f}$ representa o vetor das forças nodais requeridas para equilibrar qualquer carga concentrada ou distribuída atuando sobre o domínio computacional discretizado (Zienkiewicz \& Taylor, 2000).

Para o desenvolvimento do modelo computacional foi adotado elemento finito de casca SHELL281 (Fig. 2). Segundo ANSYS (2017), ele é adequado para análise de estruturas tipo cascas e placas que apresentem uma espessura fina ou moderada. O elemento é composto por oito nós, sendo que em cada nó há seis graus de liberdade (três de translação, nas direções $x, y, z$ e três de rotação, em torno dos eixos de $x, y, z$ ).

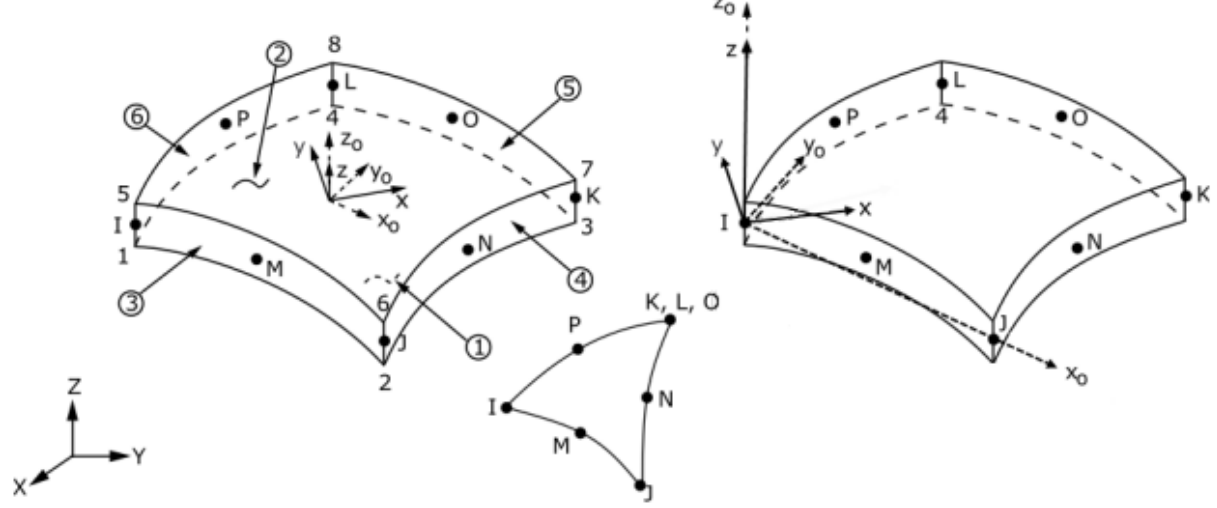

Figura 2. Ilustração do elemento de casca SHELL281 (ANSYS, 2017). 


\section{Resultados e discussões}

A verificação do modelo computacional proposto foi realizada com uma placa quadrada, engastada nos quatro lados, com dois enrijecedores ortogonais (Fig. 3) submetida a um carregamento transversal uniforme de 0,0098 $\mathrm{N} / \mathrm{mm}^{2}$. Para a placa foram consideradas as seguintes propriedades de material: um coeficiente de Poisson de 0,30 e um módulo de elasticidade de $210 \mathrm{GPa}$. A sua modelagem utilizou o elemento de casca SHELL281 do software ANSYS 18.2 versão acadêmica e teve sua verificação demonstrada em relação aos resultados numéricos obtidos por Salomon (2001).

Conforme Salomon (2001), utilizou-se uma variação na altura dos enrijecedores $h$ de 10 a $100 \mathrm{~mm}$ e a análise foi baseada nos resultados de deslocamento máximo e tensão de flexão dos enrijecedores (ou tensão na direção $x$ atuando nos enrijecedores). Para isto, inicialmente, foi desenvolvido um teste de convergência de malha para uma placa completa (ver Fig. 1(a)) e para 1/4 de placa (ver Fig. 1(b)). O teste, como exemplo, é demonstrado nas Fig. 4 e Fig. 5 para o caso em que é usada uma altura de $100 \mathrm{~mm}$.

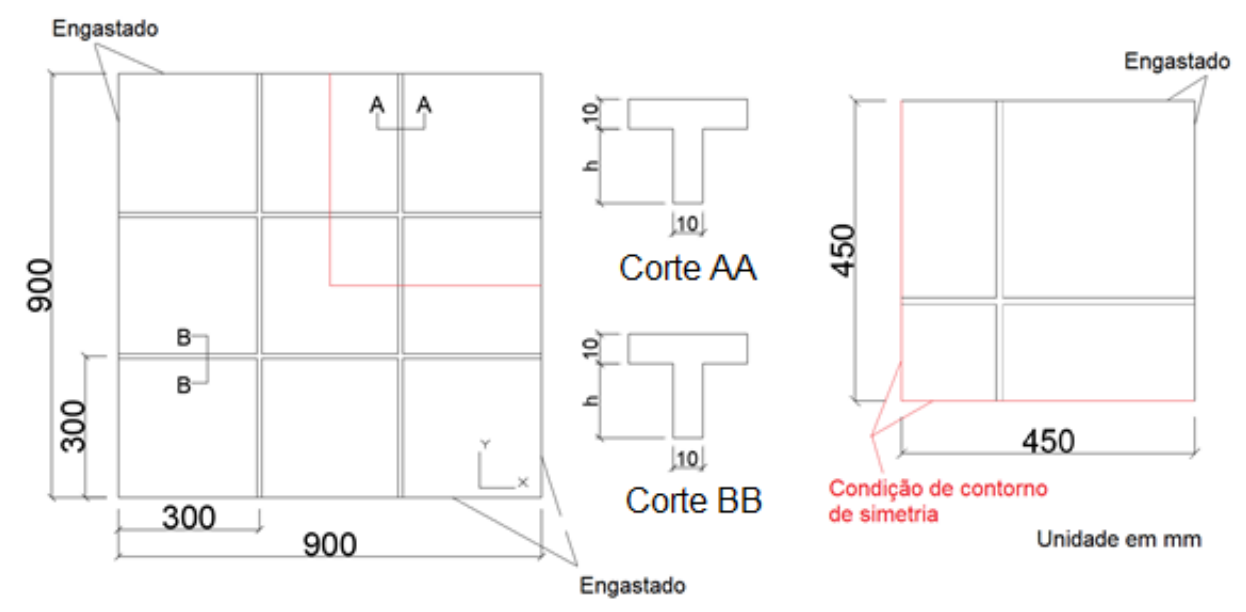

Figura 3. Placa quadrada com enrijecedores ortogonais em relação aos eixos $x$ e $y$ (Salomon, 2001).

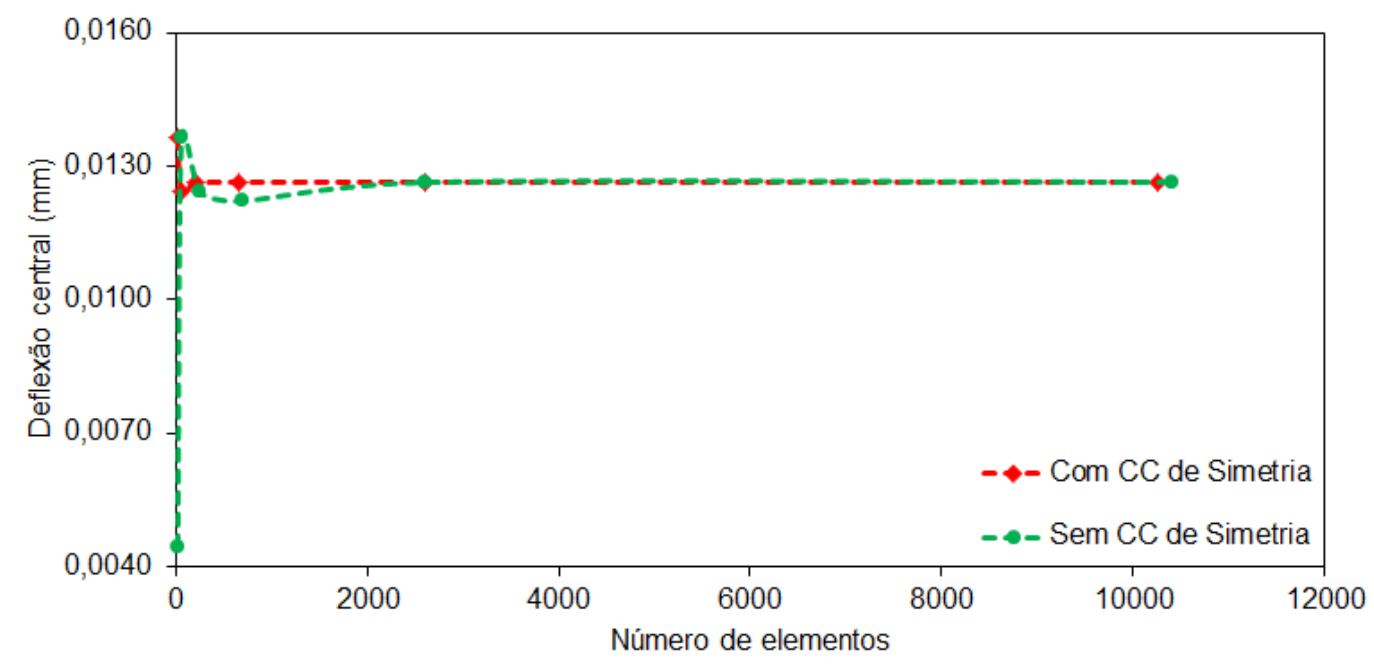

Figura 4. Testes de convergência de malha para o deslocamento máximo para $\mathrm{h}=100 \mathrm{~mm}$. 


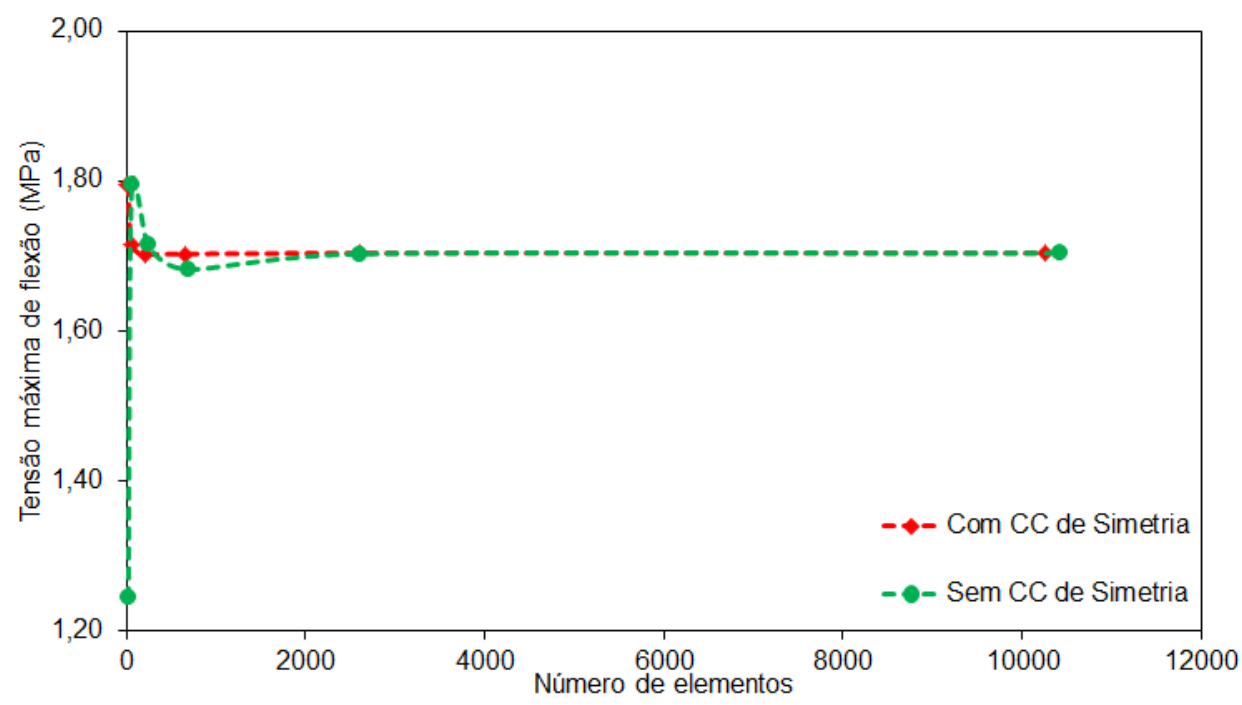

Figura 5. Testes de convergência de malha para a tensão de flexão dos enrijecedores para $h=100 \mathrm{~mm}$.

A estabilização de um teste de convergência é caracterizada como a formação de uma reta num gráfico em que ela representa a pouca variação de resultado à medida que o número de elementos aumenta. Observando a Fig. 4 a estabilização da análise da deflexão central ocorreu para a malha com 2604 elementos (tamanho de elemento de 22 $\mathrm{mm}$ ) na placa inteira e para a malha com 216 elementos (tamanho de elemento de $44 \mathrm{~mm}$ ) para a placa que emprega a condição de simetria. Com relação à análise da tensão de flexão, a Fig. 5 indica que a estabilização ocorreu para uma malha com 10416 elementos para a placa inteira e com 2604 elementos para a placa com simetria. Em ambos os casos, para a análise de tensão, o tamanho do elemento foi de $11 \mathrm{~mm}$.

Então, pode-se inferir que, ao simular $1 / 4$ da placa enrijecida através da utilização da condição de contorno de simetria, há uma diminuição no tempo de processamento sem perda de precisão. Isso ocorre em virtude da menor quantidade de elementos finitos necessários para a obtenção dos valores de deslocamento transversal (deflexão) e tensão.

Testes de convergência de malha para todos os casos $(\mathrm{h}=10 ; 20 ; 30 ; 40 ; 50 ; 60 ; 70 ; 80$; e $90 \mathrm{~mm})$ foram realizados de maneira análoga ao apresentado para o caso com $\mathrm{h}=100 \mathrm{~mm}$ (ver Figs. 4 e 5). Os resultados obtidos nos testes de convergência de malha para todos os casos considerados são então apresentados nas Tabs. 1 e 2, envolvendo a variação da altura do enrijecedor, tem seu tamanho de elemento para a placa completa na Tab. 1 e o seu equivalente com simetria na Tab. 2.

Tabela 2. Tamanho do elemento simulado para a placa completa

\begin{tabular}{ccccc}
\hline$h(\mathrm{~mm})$ & $\begin{array}{c}\text { Tamanho do } \\
\text { elemento }(\mathrm{mm})\end{array}$ & Número de elementos & $\mathrm{w}(\mathrm{mm})$ & $\sigma_{x x}$ dos enrijecedores (MPa) \\
\hline 10 & 9,50 & 9984 & 0,3287 & 14,7522 \\
20 & 9,50 & 10368 & 0,1860 & 14,1192 \\
30 & 9,75 & 10137 & 0,1004 & 10,2734 \\
40 & 9,80 & 10509 & 0,0592 & 7,2464 \\
50 & 10,00 & 9900 & 0,0387 & 5,2686 \\
60 & 10,25 & 10260 & 0,0276 & 3,9757 \\
70 & 10,50 & 10005 & 0,0211 & 3,0996 \\
80 & 10,50 & 10353 & 0,0171 & 2,4856 \\
90 & 11,00 & 10080 & 0,0145 & 2,0399 \\
100 & 11,00 & 10416 & 0,0126 & 1,7038 \\
\hline
\end{tabular}


Tabela 2. Tamanho do elemento simulado para a placa com simetria

\begin{tabular}{ccccc}
\hline$h(\mathrm{~mm})$ & $\begin{array}{c}\text { Tamanho do } \\
\text { elemento }(\mathrm{mm})\end{array}$ & Número de elementos & $\mathrm{w}(\mathrm{mm})$ & $\sigma_{x x}$ dos enrijecedores (MPa) \\
\hline 10 & 9,50 & 2496 & 0,3287 & 14,7522 \\
20 & 9,50 & 2592 & 0,1860 & 14,1192 \\
30 & 9,75 & 2688 & 0,1005 & 10,2786 \\
40 & 9,80 & 2784 & 0,0592 & 7,2502 \\
50 & 10,00 & 2475 & 0,0387 & 5,2686 \\
60 & 10,25 & 2565 & 0,0276 & 3,9757 \\
70 & 10,50 & 2655 & 0,0212 & 3,1018 \\
80 & 10,50 & 2745 & 0,0171 & 2,4874 \\
90 & 11,00 & 2520 & 0,0145 & 2,0399 \\
100 & 11,00 & 2604 & 0,0126 & 1,7038 \\
\hline
\end{tabular}

Após a realização dos testes de convergência de malha, os resultados numéricos independentes de malha obtidos no presente estudo foram comparados aos apresentados por Salomon (2001), visando realizar a verificação do modelo computacional proposto.

Com relação aos modelos numéricos abordados por Salomon (2001), eles foram baseados nos elementos de viga Hermitiano combinado com elementos de casca com quatro nós; apenas elementos de casca com oito nós; e, elementos tridimensionais com vinte e sete nós, utilizado como referência aos demais. Dentre estes modelos, duas modificações foram propostas. A primeira alterou a excentricidade dos enrijecedores do elemento Hermitiano devido à deflexão ocorrer em torno da superfície média da placa, e não sobre o eixo neutro da seção transversal. Já, a segunda modificação, tratou a placa e o enrijecedor como uma única estrutura por causa da sobreposição de superfícies que ocorria entre a placa e o enrijecedor quando utilizando apenas elementos de casca. Além disso, duas abordagens foram apresentadas envolvendo os elementos de casca. A primeira considera o acréscimo de meia espessura de placa na altura dos enrijecedores (elemento de casca rígido); e, a segunda, faz com que o enrijecedor chegue até a metade da placa, causando sobreposição de estrutura e perda de resistência (elemento de casca flexível).

Portanto, na Fig. 6 são comparados os resultados para a deflexão central obtido no presente estudo utilizando condição de contorno de simetria (1/4 de placa) com os de Salomon (2001). Da mesma maneira, na Fig. 7 os resultados de tensão máxima são mostrados.

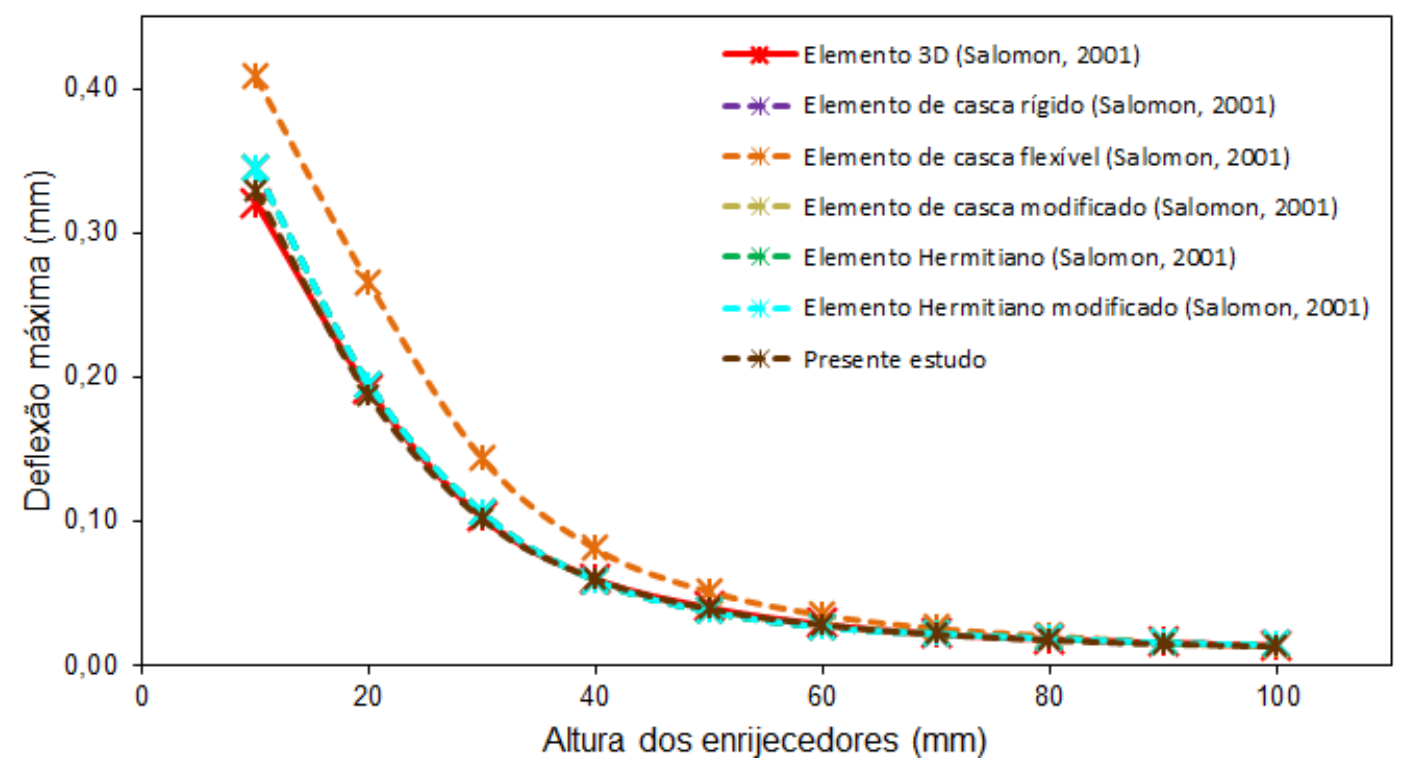

Figura 6. Comparativo dos resultados numéricos de deflexão máxima. 


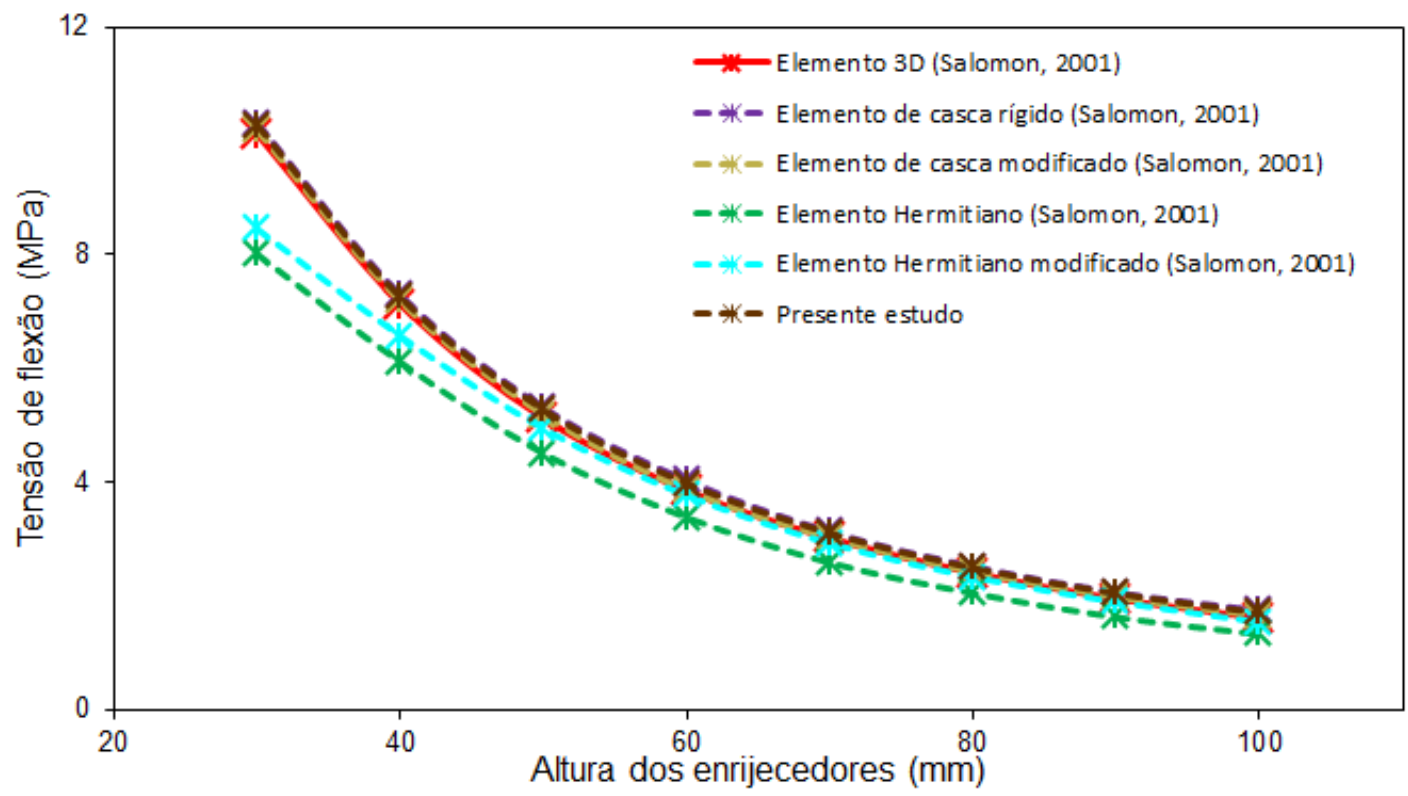

Figura 7. Comparativo dos resultados numéricos da tensão de flexão dos enrijecedores.

As Figs. 6 e 7 indicam que os resultados de deflexão e tensão, respectivamente, obtidos no presente estudo possuem boa concordância com os resultados apresentados por Salomon (2001). Dentre os modelos computacionais adotados em seu estudo o mais preciso é o com elemento 3D. Então, usando o modelo 3D como referência, pode-se constatar uma precisão de $99,41 \%$ para a altura de $40 \mathrm{~mm}$, e $93,22 \%$ para a altura de $90 \mathrm{~mm}$. Já, para a análise da tensão de flexão, o modelo de casca flexível não foi utilizado pelo autor. Logo, a comparação entre o modelo SHELL281 (Presente estudo) e o modelo 3D, constatou-se uma variação de tensão de 5,35\% para a altura de $100 \mathrm{~mm}$.

Por fim, para realizar uma avaliação qualitativa em relação à distribuição de deflexões (Fig. 8) e de tensões (Fig. 9), os resultados obtidos com o modelo computacional com condição de contorno de simetria foram comparados aos obtidos com o modelo que considera a placa inteira. Para isso, levando em conta a Fig. 3, os casos com $h=10 \mathrm{~mm}$ e $h$ $=100 \mathrm{~mm}$ foram empregados. Com relação as Fig. 8 e Fig. 9, encontram-se a esquerda os modelos computacionais das placas completa e a direita os modelos que empregaram a condição de contorno de simetria.

Observando as figuras, é notado que as placas que utilizam a simetria demonstram uma boa semelhança na sua forma defletida e na distribuição de tensão com os modelos de placa completa. Também, é notado que a variação da altura dos enrijecedores nas placas apresenta certo padrão na distribuição dos deslocamentos e das tensões. Por exemplo, a placa com $h=10 \mathrm{~mm}$ tem o comportamento de deflexão e tensão mais global no centro da placa. A sua forma defletida é bastante parecida à de uma placa que não contém nenhum enrijecedor em sua estrutura, conforme pode ser visualizado na Fig. 8. Enquanto que a placa com $h=100 \mathrm{~mm}$ tem regiões confinadas entre os enrijecedores que se comportam como pequenas placas totalmente engastadas nas quatro bordas. Demonstrando, como esperado, uma maior rigidez estrutural pela maior influência dos enrijecedores e uma melhor distribuição de tensão de flexão ao longo de sua estrutura, conforme pode ser observado na Fig. 9. 


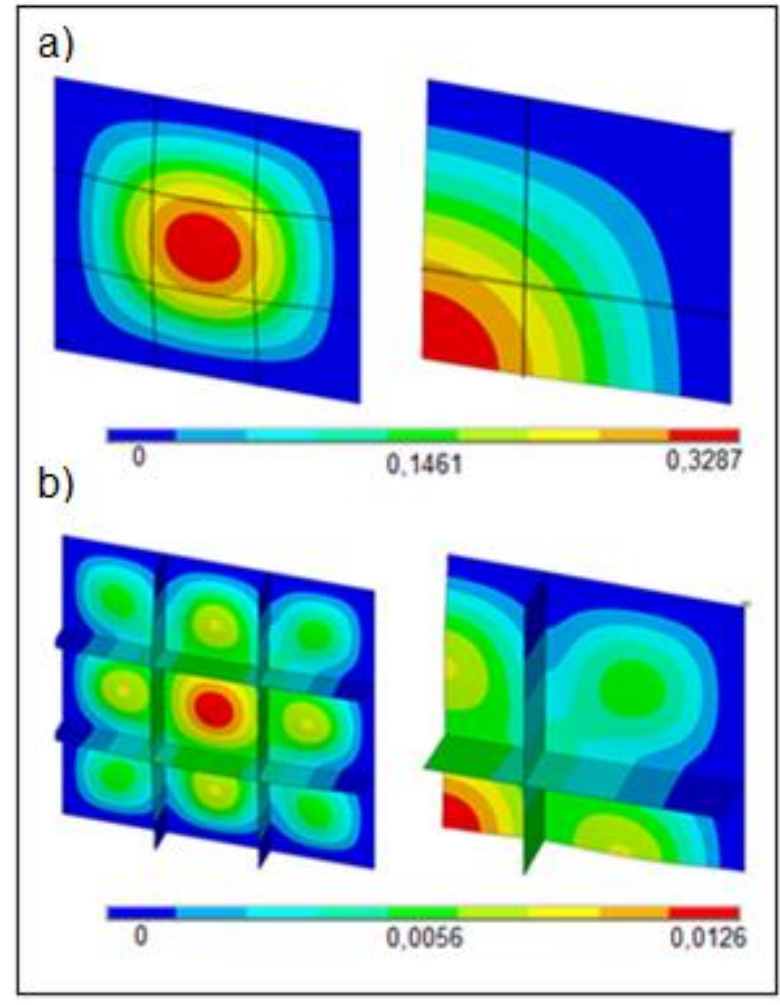

Figura 8. Distribuições de deflexões na placa enrijecida inteira e com simetria com a) $h=10 \mathrm{~mm}$ e b) $h=100 \mathrm{~mm}$.

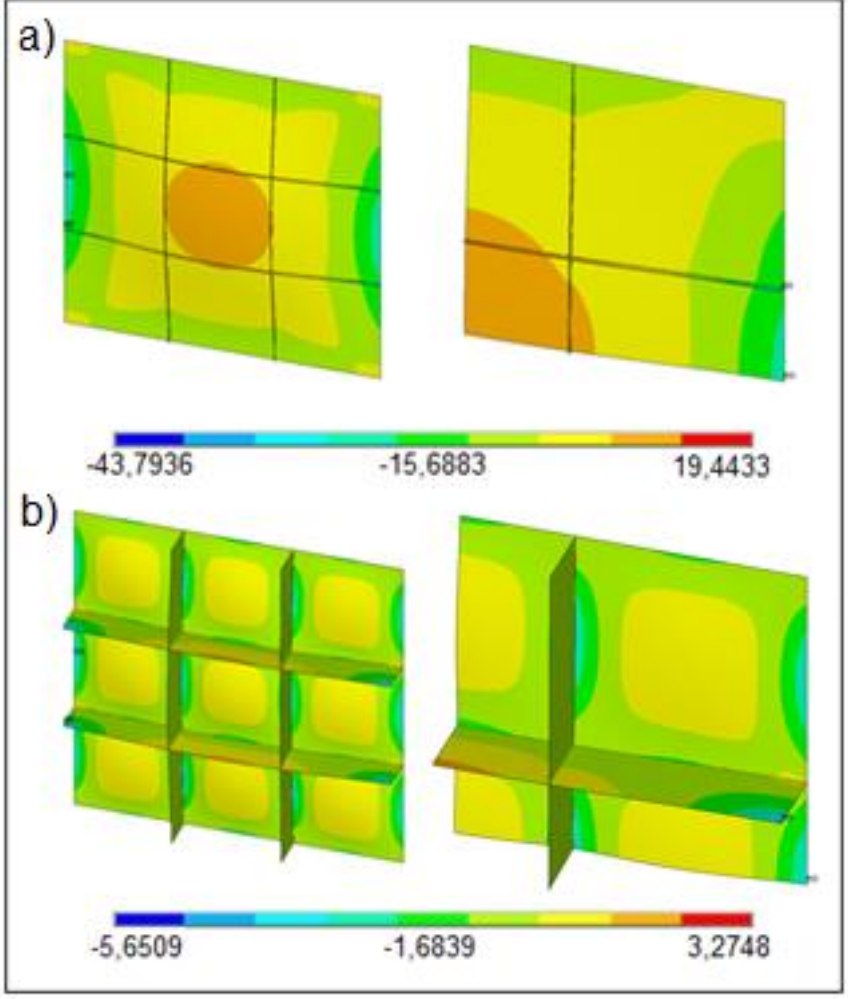

Figura 9. Distribuições de tensões na placa enrijecida inteira e com simetria com a) $h=10 \mathrm{~mm}$ e b) $h=100$ $\mathrm{mm}$.

\section{Conclusão}

O presente artigo apresentou simulações numéricas de comportamentos mecânicos de placas enrijecidas submetidas a um carregamento transversal. Os modelos computacionais empregaram a condição de contorno de simetria e foram baseados no elemento finito SHELL281 do software ANSYS 18.2. Os resultados de deslocamento e tensão de flexão obtida com o modelo computacional proposto foram comparados com resultados obtidos por diferentes modelos numéricos usados em Salomon (2001).

O trabalho possibilitou determinar um ganho de tempo no processamento computacional das simulações que utilizam a condição de contorno de simetria em $1 / 4$ de placa, porque elas reduzem o domínio computacional do problema e, como consequência, necessitam de menos elementos finitos para sua discretização espacial. Notou-se também que a condição de contorno de simetria possibilita a utilização de malhas mais refinadas com relação às simulações que consideram a placa inteira no software ANSYS versão acadêmica. Esse é um aspecto importante, uma vez que a versão acadêmica do ANSYS possui uma limitação em relação o refinamento de malha. Com relação à precisão, os valores obtidos foram coerentes em relação às simulações envolvendo a placa completa e com os modelos apresentados em Salomon (2001), demonstrando que a metodologia empregada é adequada para a determinação das deflexões e tensões. Portanto, é possível considerar verificado o modelo computacional que emprega condição de contorno de simetria.

Por fim, possibilita-se a utilização do modelo computacional verificado para futuros estudos sobre o comportamento mecânico de placas enrijecidas.

Agradecimentos: Os autores agradecem ao CNPq, CAPES e FAPERGS pelo apoio financeiro.

\section{References}

1. ANSYS, Inc. ANSYS ${ }^{\circledR}$ Academic Research Mechanical, Release 18.2, Help System, Coupled Field Analysis Guide.

2. Bellei, I. H. (1998). Edifícios Industriais em aço (2a ed.). São Paulo.

3. Rossowt, M. P., Ibrahimkhail, A. K. (1978). Constraint Method Analysis of Stiffened Plates. Computers \& Structures, Vol. 8, pp 51-60. 
4. https://doi.org/10.1016/0045-7949(78)90159-1

5. Salomon, A. (2001). An evaluation of finite element models of stiffened plates (dissertação de mestrado). Retirado de Massachusetts Institute of Technology.

6. Stolarski, T.; Nakasone, Y.; Yoshimoto, S. (2018). Engineering Analysis with ANSYS Software (2nd ed.). The Boulevard, Langford Lane, Kidlington, Oxford OX5 1GB, United Kingdom.

7. Szilard, R. (2004). Theories and applications of plate analysis: classical, numerical and engineering methods. Hoboken: New Jersey.

8. Troina, G. da S. (2017). Modelagem computacional e Método Design Construtal aplicados à otimização geométrica de placas finas de aço com enrijecedores submetidas a carregamento transversal uniforme (dissertação de mestrado). Retirado de Universidade Federal do Rio Grande.

9. Vaz, L. E. (2011). Método dos elementos finitos em análise de estruturas (1 1 a ed.). Rio de Janeiro.

10. Vellasco et al. (2014). Modelagem de estruturas de aço e mistas (1a ed.). Rio de Janeiro.

11. Ventsel, E., Krauthammer, T. (2001). Thin plates and shells: Theory, Analysis and Applications (1st ed.). New York: Marcel Dekker.

12. Zienkiewicz, O. C., Taylor, R. L. (2000). The Finite Element Method - Volume 1: The basis. Butterworth-Heinemann, Oxford. 